\title{
Versorgungsstrukturgesetz
}

\section{Wohl und Wehe der Regionalisierung}

\author{
Das Versorgungsstrukturgesetz soll mittels Regionalisierung unter anderem Bürokratie abbauen. \\ Bei einer Analyse der Pläne der schwarz-gelben Regierungskoalition offenbaren sich aus der Sicht \\ der Fachärzte jedoch zahlreiche Risiken und Nebenwirkungen.
}

$\mathrm{D}$ er zentrale Punkt des Versorgungsstrukturgesetzes (GKVVSG) ist für die Fachärzte der Aufbau einer ambulanten spezialärztlichen Versorgung unabhängig von und in Konkurrenz mit der allgemeinen fachärttlichen Versorgung. Dabei werden die Fachärzte in einen Wettbewerb mit den Krankenhäusern gedrängt. Inzwischen gibt es massive Kritik der fachärztlichen Berufsverbände an diesem Vorhaben. Die systematische Trennung der fachärztlichen Versorgung in verschiedene Ebenen wird dabei als Spaltpilz für die Fachärzte gesehen. Das ambulante Leistungsspektrum der Fachärzte sei überwiegend sowohl auf Versorgungsfälle als auch auf Spezialleistungen im Sinne der $\S \S 115 \mathrm{~b}$ und 116 SGB V ausgerichtet, somit einer krankenhausnahen Leistungserbringung, stellt die Gemeinschaft fachärztlicher Berufsverbände (GFB) fest.

\section{Zahlreiche Nachbesserungen gefordert}

Bei den Kassenärztlichen Vereinigungen (KVen) formiert sich inzwischen der $\mathrm{Wi}$ derstand gegen die Pläne des Bundesgesundheitsministers. Eine Resolution der Vertreterversammlung der KV Niedersachsen findet breite Unterstützung. Gefordert werden folgende Nachbesserungen im Gesetzesentwurf:

- Der Krankenhaus-Sektor müsse an der

Finanzierung zu gleichen Bedingungen beteiligt werden. Es dürfe keine Finanzierung der Vergütung spezialärztlicher Leistungen durch eine Bereinigung der Gesamtvergütung der Vertragsärzte geben.

_ Eine Öffnung der Krankenhäuser für ambulante spezialärztliche Leistungen sei erst dann möglich, wenn eine einheitlich betriebswirtschaftlich kalku- lierte Vergütung unter Berücksichtigung der Investitionskosten umgesetzt ist. Der geplante Investitionsabschlag von $5 \%$ für die Krankenhäuser sei völlig unzureichend.

_ Die neue Regelung solle auf seltene Erkrankungen (fünf Erkrankungen pro eine Million Einwohner) und bestehende Versorgungsdefizite eingegrenzt werden.

_Die Einzelleistungsvergütung zu festen

Preisen ohne Mengenbegrenzung sei unerlässlich.

— Grundsätzlich soll über die KVen abgerechnet werden.

_ Die KVen sollen bei der Festlegung der Qualitätsstandards und der Indikationsliste für die spezialärztliche Versorgung beteiligt werden.

— Die Qualifikationsanforderungen aus dem ambulanten Bereich müssten auch für die Krankenhäuser mit voller Verbindlichkeit gelten. Der Facharzt-Standard sei gesetzlich festzuschreiben.

\section{Regionalisierung nicht zwingend von Vorteil}

Auch der Verzicht auf die allgemeinen Kodierrichtlinien ist nur auf den ersten Blick von Vorteil. Bundesgesundheitsminister Daniel Bahr feiert diesen Bürokratieabbau als Erfolg für die Ärzte. Tatsächlich wird das „Bürokratiemonster“ aber nur auf der Bundesebene erfolgreich verjagt. Auf regionaler Ebene werden die Ärzte sehr rasch wieder mit der Forderung der Krankenkassen konfrontiert werden, Diagnosen differenziert zu kodieren, sobald sich die morbiditätsbedingte Gesamtvergütung ändert. Modellrechnungen legen nahe, dass die Veränderung der Morbidität bei den Zahlungen der Krankenkassen im Sinne der Ärzte angemessen berücksichtigt wird.

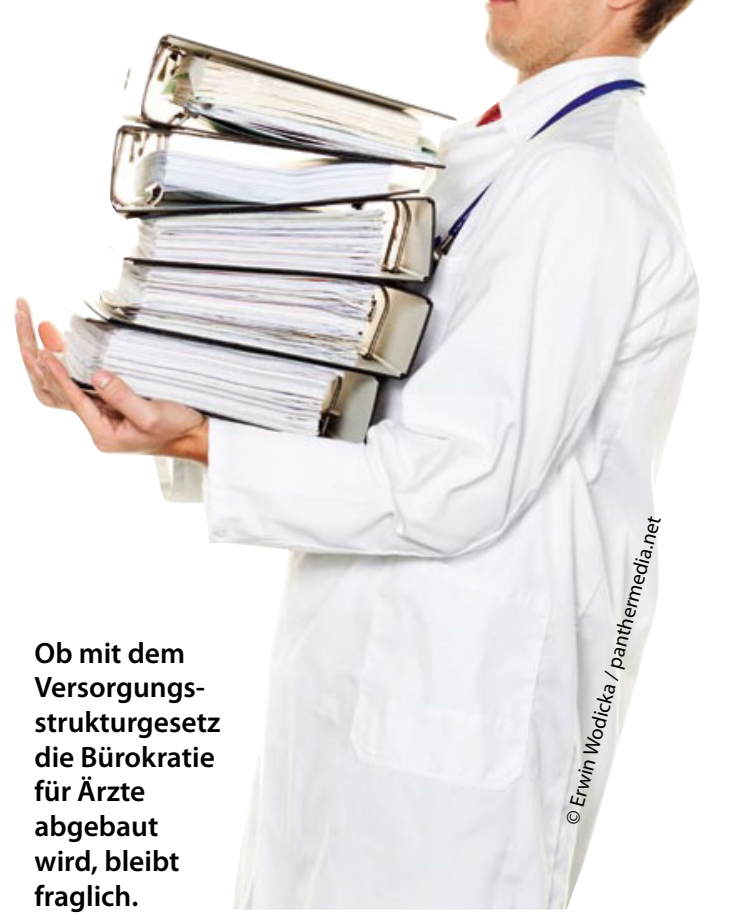

Auch die Regionalisierung der Vertragsverhandlungen mit den Krankenkassen bedarf einer kritischen Bewertung. So manchen Facharzt treibt die Sorge um, dass die Verhandlungen der regionalen KV-Fürsten weniger erfolgreich sein werden als die Verhandlungen auf der Bundesebene unter der Verantwortung der Kassenärztlichen Bundesvereinigung (KBV). Die Regionalisierung könnte sich auch als Rückschlag für Fachärzte in Regionen erweisen, die mit unterdurchschnittlichen Vergütungen konfrontiert sind. „Gleiches Geld für gleiche Leistungen“, diese Forderung wird auf Dauer ins Hintertreffen geraten, wenn die unterschiedliche Finanzkraft in den einzelnen Bundesländern auf die Veränderung der Gesamtvergütungen und die Vergütung in den regionalen Euro-Gebührenordnungen ausstrahlt. Norbert Fischer 